\title{
Exact time autocorrelation function of the $N$-spin classical Heisenberg equivalent neighbor model
}

\author{
Richard A. Klemm and Marco Amedurif \\ Max-Planck-Institut für Physik komplexer Systeme, \\ Nöthnitzer Straße 38, D-01187 Dresden, Germany
}

(Dated: October 25, 2018)

\begin{abstract}
We reduce the autocorrelation function $\mathcal{C}_{11}(t)$ of the equivalent neighbor model of $N$ classical spins exhibiting Heisenberg dynamics and exchange coupling $J$ to quadrature. As the temperature $T \rightarrow \infty, \mathcal{C}_{11}(t) \propto t^{-N}$ for $J t>>1$. At low $T$, the antiferromagnetic $\mathcal{C}_{11}(t)$ is a simple function of $(J T)^{1 / 2} t$, exhibiting strong frustration, but the ferromagnetic $\mathcal{C}_{11}(t)$ oscillates in a single mode the frequency of which approaches $N J$ as $T \rightarrow 0$. We conjecture that as $T \rightarrow \infty$, the near-neighbor correlation functions of $N$-spin classical Heisenberg rings are simply obtained from these results.

PACS numbers: 05.20.-y, 75.10.Hk, 75.75.+a, 05.45.-a
\end{abstract}

Recently, there has been a considerable interest in the physics of magnetic molecules. [1] These consist of small clusters of magnetic ions imbedded within a nonmagnetic ligand group, which may crystalize into large, well-ordered single crystals of sufficient quality for neutron scattering studies. Each type of magnetic molecule is characterized not only by the number $N$ of spins in the molecule, but also by the spatial configuration of the spins. Many examples of rings exist, [2, 3] but there are also examples of denser clusters. [1, 1 , Usually, the spins interact mainly via the Heisenberg exchange interaction. Although interest in the dynamics of Heisenberg spin rings has been strong for many years, most of the work involved numerical simulation of the two-spin correlation functions. Recently, however, exact results for the Heisenberg dimer, isosceles and equilateral triangle, and four-spin ring were presented, [5, 6, 7] but larger rings can not be solved using this technique.

The equivalent neighbor model is the simplest model for the dynamics of a nanomagnet, first proposed by Kittel and Shore. [8] In this model, every spin interacts equally with all of the others. Although the Heisenberg dynamics of the $N \rightarrow \infty$ limit at infinite temperature $T$ were obtained previously, [9] they have not yet been solved for $5 \leq N<\infty$. Although this model is oversimplified for direct comparision with experiment when $N \geq 5$, its exact solution could serve as a benchmark for future theoretical and experimental studies.

In this letter, we present an exact single integral representation of the autocorrelation function $\mathcal{C}_{11}(t)$ for the equivalent neighbor model with arbitrary $N$ and $T$, both for ferromagnetic (FM) and antiferromagnetic (AFM) exchange couplings. As $T \rightarrow \infty$, an accurate asymptotic $1 / N$ expansion is found. As $T \rightarrow 0$, the $\mathrm{AFM} \mathcal{C}_{11}(t)$ reduces exactly to a simple function of $(J T)^{1 / 2} t$ for arbitrary $N$. For the FM case at low $T, \mathcal{C}_{11}(t)$ oscillates in a single mode with a frequency that approaches $N J$ as $T \rightarrow 0$, and a shape that fits a scaling relation. From additional studies of three- and four-spin rings with various amounts of bridging spins that interact only with the ring spins, we conjecture that the long-time $T \rightarrow \infty$ asymptotic limit values of the near-neighbor correlation functions for equivalent neighbor coupling and near-neighbor coupling in classical Heisenberg $N$-spin rings are identical.

The Hamiltonian of the $N$-spin classical Heisenberg equivalent neighbor model is $H=-J s^{2} / 2$, where the total spin $\mathbf{s}=\sum_{i=1}^{N} \mathbf{s}_{i}(t)$ is a constant. The dynamics are given by $d \mathbf{s}_{i}(t) / d t=J \mathbf{s}_{i} \times \mathbf{s}$, where $s_{i}=\left|\mathbf{s}_{i}\right|=1$. $\mathbf{s}_{i}(t)$ may be written as $\mathbf{s}_{1}(t)=s_{1,||} \hat{\mathbf{s}}+s_{1, \perp}\left[\hat{\mathbf{x}} \cos \left(s t^{*}\right)-\right.$ $\left.\hat{\mathbf{y}} \sin \left(s t^{*}\right)\right]$, where $t^{*}=J t, \hat{\mathbf{s}}=\hat{\mathbf{x}} \times \hat{\mathbf{y}}, s_{1, \|}(s, x)=\left(s^{2}+\right.$ $\left.1-x^{2}\right) /(2 s), x=\left|\mathbf{s}-\mathbf{s}_{1}\right|$, and $s_{1, \|}^{2}+s_{1, \perp}^{2}=1$. Letting $\alpha=J /\left(2 k_{B} T\right)$, where $k_{B}$ is Boltzmann's constant, the autocorrelation function $\mathcal{C}_{11}(t)=\left\langle\mathbf{s}_{1}(t) \cdot \mathbf{s}_{1}(0)\right\rangle$ may be written

$$
\begin{aligned}
\mathcal{C}_{11}(t)= & \frac{1}{Z_{N}} \int_{0}^{N-1} \mathcal{D}_{N-1}(x) d x \int_{|x-1|}^{x+1} s e^{\alpha s^{2}} d s \times \\
& \times\left[s_{1, \|}^{2}(s, x)+\cos \left(s t^{*}\right) s_{1, \perp}^{2}(s, x)\right],
\end{aligned}
$$

where the partition function $Z_{N}=\int_{0}^{N} 2 s \mathcal{D}_{N}(s) e^{\alpha s^{2}} d s$ and $\mathcal{D}_{N}(s)$ is the $N$-spin density of states. $\mathcal{D}_{N}(x)$ is piecewise continuous,

$$
\begin{aligned}
\mathcal{D}_{N}(x)= & \Theta(x) \sum_{p=0}^{E[(N-1) / 2]} \Theta(N-2 p-x) \times \\
& \times \Theta(x-N+2 p+2) d_{N-2 p}(x), \\
d_{N-2 p}(x)= & \sum_{k=0}^{p} \frac{(-1)^{k}(N-2 k-x)^{N-2}}{2^{N-1}(N-2) !}\left(\begin{array}{c}
N \\
k
\end{array}\right),
\end{aligned}
$$

where $\Theta(x)$ is the Heaviside step function, $\left(\begin{array}{c}n \\ m\end{array}\right)$ are binomial distribution coefficients, and $E(x)$ is the largest integer in $x$. The near-neighbor correlation function $\mathcal{C}_{12}(t)=\left\langle\mathbf{s}_{1}(t) \cdot \mathbf{s}_{2}(0)\right\rangle$ is simply obtained from $\mathcal{C}_{11}(t)$ and the sum rule $\left\langle s^{2}\right\rangle / N=1=\mathcal{C}_{11}(t)+(N-1) \mathcal{C}_{12}(t)$.

It is useful to write $\delta \mathcal{C}_{11}(t)=\mathcal{C}_{11}(t)-\lim _{t \rightarrow \infty} \mathcal{C}_{11}(t)$. Since $\mathcal{C}_{11}(0)=1$, it suffices to obtain $\delta \mathcal{C}_{11}(t)$ for all $t$. Af- 
ter some algebra, we have reduced $\delta \mathcal{C}_{11}(t)$ to quadrature,

$$
\begin{aligned}
\delta \mathcal{C}_{11}(t)= & \sum_{p=0}^{E[(N-1) / 2]} \int_{N-2 p-2}^{N-2 p} d s \cos \left(s t^{*}\right) f_{N-2 p}(s),(4) \\
f_{N-2 p}(s)= & \Theta(s) \frac{e^{\alpha s^{2}}}{Z_{N}} \sum_{k=0}^{p}(N-2 k-s)^{N-1} g_{k}(s) \\
g_{0}(s)= & \frac{(N-1) a(s)}{2^{N-3} s(N+2) !}, \\
a(s)= & N(s+1)^{3}-N^{2}(s+1)+N+s^{3}-2 s,(7) \\
g_{k}(s)= & \frac{(-1)^{k} b_{N-2 k}(s)}{s 2^{N-3} N(N+2) !}\left(\begin{array}{c}
N \\
k
\end{array}\right), k \neq 0 \\
b_{m}(s)= & s^{2} N(N-1)[3 m+s(N+1)] \\
& +[s(N-1)+m] \times \\
& \times\left[6 m^{2}-N(N+1)(N+2)\right]
\end{aligned}
$$

To obtain the long-time asymptotic behavior for $|\alpha| \ll$ 1, we integrate Eq. (4) by parts $N-1$ times. We find

$$
\begin{aligned}
\delta \mathcal{C}_{11}(t) \underset{t * \gg 1}{\sim} & \sum_{p=0}^{E(N / 2)} h_{p} \cos \left[(N-2 p)\left(t^{*}-\pi / 2\right)\right] \times \\
& \times\left(t^{*}\right)^{-N} e^{(N-2 p)^{2} \alpha} / Z_{N} \\
h_{p}= & \frac{\left[(N-2 p)^{2}-N\right]}{2^{N-3} N}\left(\begin{array}{c}
N \\
p
\end{array}\right) \times \\
& \times\left[1+(p-1) \delta_{p, N / 2}\right]
\end{aligned}
$$

For $N=M^{2}$, one of the terms in Eq. (10) vanishes, as for the four-spin ring. [6] More important, the leading long-time behavior $\propto 1 / t^{* N}$, which falls off very rapidly for large $N$. Although Eq. (10) fails for $|\alpha| \gg 1$, its behavior for $\alpha \neq 0$ is useful to illuminate the dramatic difference between the long-time asymptotic behavior for the FM and AFM cases. For the FM case, $\alpha>0$, the dominant behavior $\propto \cos \left[N\left(t^{*}-\pi / 2\right)\right]$, as all of the spins oscillate together. In the AFM case, $\alpha<0$, the dominant long-time asymptotic behavior is given by the smallest possible oscillation frequency. As shown in the following, $\mathcal{C}_{11}(t)$ is always finite as $T \rightarrow 0$.

As $T, N \rightarrow \infty$, Liu and Müller obtained an analytic form for $\mathcal{C}_{11}$, but their procedure was not extendable to finite $N$. 9. From the integral representation, $\mathcal{D}_{N}(x)=$ $\int_{0}^{\infty} 2 p^{1-N} \sin ^{N} p \sin (p x) d p / \pi$, we expand $\sin ^{N} p / p^{N}$ as $\exp \left[-N\left(p^{2} / 6+p^{4} / 180+p^{6} / 2835+\ldots\right)\right]$ to obtain a $1 / N$ asymptotic expansion for $\mathcal{C}_{11}(t)$ as $T \rightarrow \infty$,

$$
\begin{aligned}
\lim _{\substack{T \rightarrow \infty \\
N \gg 1}} \mathcal{C}_{11}(t) \sim & {\left[1+2 e^{-\tilde{t}^{2}}\left(1-2 \tilde{t}^{2}\right)\right] / 3 } \\
+ & 2\left[1-e^{-\tilde{t}^{2}}\left(1+\tilde{t}^{2}-\tilde{t}^{4} / 3-2 \tilde{t}^{6}\right)\right] /(5 N) \\
+ & \frac{12}{175 N^{2}}\left[1-e^{-\tilde{t}^{2}}\left(1+\tilde{t}^{2}-\frac{157}{36} \tilde{t}^{4}+\right.\right. \\
& \left.\left.\frac{50}{27} \tilde{t}^{6}-\frac{97}{108} \tilde{t}^{8}+\frac{7}{18} \tilde{t}^{10}\right)\right],
\end{aligned}
$$

where $\tilde{t}=J t[(N-1) / 6]^{1 / 2}$. From Eq. (12), $\mathcal{C}_{11}(0)=1$ through order $1 / N^{2}$, as required, and $\mathcal{C}_{11}(t)$ has the correct $N \rightarrow \infty$ limit. [9] However, Eq. (12) is highly accurate for intermediate $N$ values as well. In Fig. 1, we have plotted $\lim _{T \rightarrow \infty} \mathcal{C}_{11}(t)$ for $N=5,6,7,8,9,25,50,100, \infty$ as a function of $J t[(N-1) / 6]^{1 / 2}$. The curves for $N=$ $25,50,100, \infty$ were obtained from Eq. (12), whereas the other curves were generated from the exact solution, Eqs. (4)-(9). Equation (12) is very accurate at short and long times, even for $N=5$. We note that for $\tilde{t} \lesssim 1$, $\lim _{T \rightarrow \infty} \mathcal{C}_{11}(t)$ is a universal function of $J t \sqrt{N-1}$, as suggested by the $1 / N$ expansion. Although the $N \rightarrow \infty$ curve is exact, and the curves for $N=50,100$ are quite accurate, the curve for $N=25$ exhibits a small $(\approx 1 \%)$ inaccuracy for $1.2 \lesssim \tilde{t} \lesssim 2.2$.

At arbitrary $N, t$ and $N \gg 1, t \rightarrow \infty$, respectively,

$$
\begin{aligned}
& \lim _{T \rightarrow \infty} \mathcal{C}_{11}(t)=1 / N+(N-1)\left[\delta_{N}+f_{N}(t)\right] \\
& \lim _{t, T \rightarrow \infty} \mathcal{C}_{11}(t) \underset{N \gg 1}{\sim} \frac{1}{3}+\frac{2}{5 N}+\frac{12}{175 N^{2}} \\
& \delta_{N} \underset{N \gg 1}{\sim} \frac{175 N^{2}-315 N+36}{525 N^{2}(N-1)}
\end{aligned}
$$

In the inset of Fig. 1, we have compared the exact values of $\delta_{N}$ with those obtained from Eq. (15) for $3 \leq N \leq 65$. For $N=3$, the differences are noticeable on this curve. As $N$ increases from 5 to 65 , the relative difference decreases rapidly from $0.14 \%$ to $0.2 \mathrm{ppm}$. Since the exact formula for $\delta_{N}$ with $N$ odd (even) contains non-trivial contributions of logarithms of all prime numbers from 3 to $N(2$ to $N / 2)$, respectively, the extremely good numerical agreement between the exact $\delta_{N}$ and Eq. (15) is remarkable.

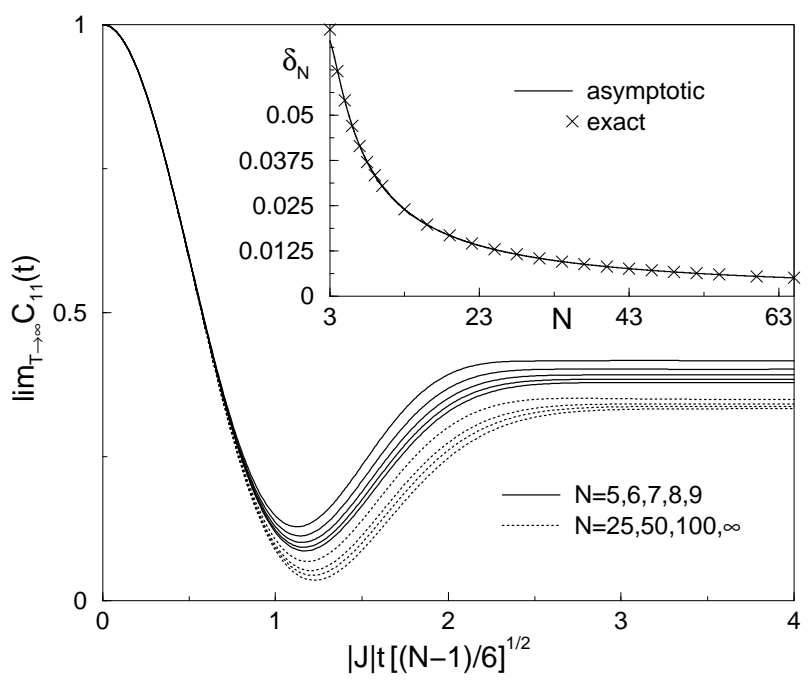

FIG. 1: Plots of $\lim _{T \rightarrow \infty} \mathcal{C}_{11}(t)$ versus $|J| t[(N-1) / 6]^{1 / 2}$, for $N=5,6,7,8,9$ from Eqs. (4)-(9) (solid), and for $N=$ 25, 50,100, $\infty$ from Eq. (12) (dashed). Inset: Plot of the exact $(\times)$ and asymptotic values, Eq. (15), of $\delta_{N}$. 


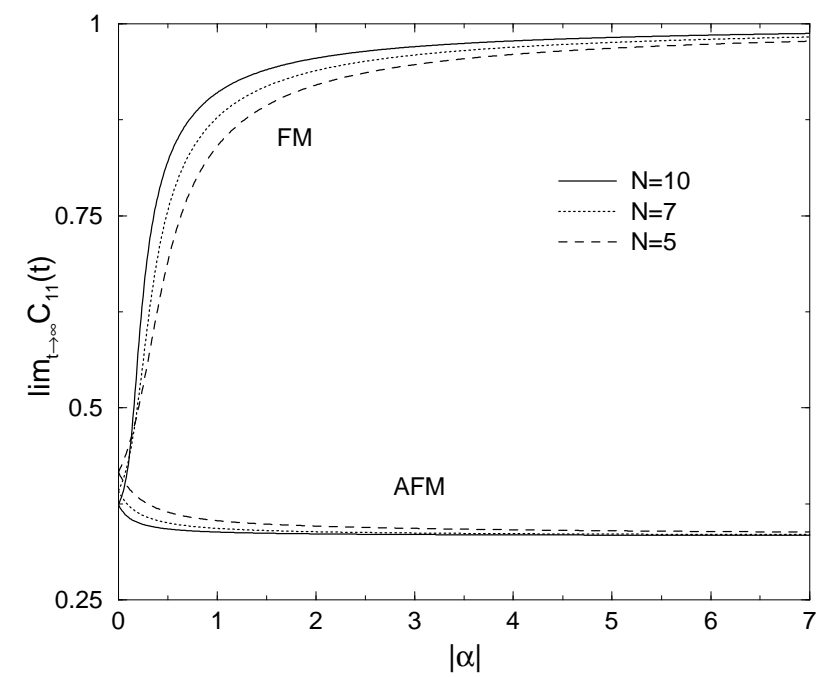

FIG. 2: Plots of $\lim _{t \rightarrow \infty} \mathcal{C}_{11}(t)$ versus $|\alpha|$ for $N=5,7,10$ and for both ferromagnetic and antiferromagnetic couplings.

In Fig. 2, we plot the long-time asymptotic limits $\lim _{t \rightarrow \infty} \mathcal{C}_{11}(t)$ for both the FM and AFM cases, for $N=5,7,10$, as functions of $|\alpha|$. The FM values approach 1 as $T \rightarrow 0$. But, the low- $T$ AFM values rapidly approach their $T=0$ asymptotic limit, $\frac{1}{3}$. This indicates the spins are strongly frustrated, since one might naively expect the AFM $\lim _{t \rightarrow \infty} \mathcal{C}_{11}(t)=0$, as for the four-spin ring. [6] In that case, the unfrustrated alternating spin direction configuration was possible. In this AFM case, however, the $N \geq 3$ spins are as frustrated as $T \rightarrow 0$ as are an infinite number of them as $T \rightarrow \infty$.

We now examine the low- $T$ AFM dynamics. In this case, the dominant contibutions to the numerator of $\mathcal{C}_{11}$ and to $Z_{N}$ arise from $s \ll 1$. The leading contributions to these integrals are $P_{N} s^{2} e^{-|\alpha| s^{2}}$ and $Q_{N} s^{2} e^{-|\alpha| s^{2}}$, respectively, where $P_{N}$ and $Q_{N}$ are complicated functions of $N$, but for arbitrary $N \geq 3, P_{N} / Q_{N}=\frac{2}{3}$. Thus, as $T \rightarrow 0$, the Fourier transform $\delta \mathcal{C}_{11}(\omega)$ of $\delta \mathcal{C}_{11}(t)$ attains the exact uniform asymptotic form,

$$
|\alpha|^{-1 / 2} \delta \mathcal{C}_{11}(\omega) \underset{T \rightarrow 0}{\sim} \frac{8}{3 \sqrt{\pi}} \tilde{\omega}^{2} e^{-\tilde{\omega}^{2}}
$$

where $\tilde{\omega}=|\alpha|^{-1 / 2} \omega /|J|$. For $N \geq 5$, corrections to Eq. (16) are of $\mathcal{O}\left(|\alpha|^{-1}\right)$, whereas for $N=4$, they are of $\mathcal{O}\left(|\alpha|^{-1 / 2}\right)$. In Fig. 3, we show the frequency dependence of the low- $T$ AFM mode. Since $\mathcal{C}_{11}(t)$ for fixed $N$ is a uniform function of $|J| t /|\alpha|^{1 / 2}$, the amplitude of $\delta \mathcal{C}_{11}(\omega)$ must also be scaled by $|\alpha|^{-1 / 2}$ for the curves to nearly coincide. Here we plot the curves at $\alpha=-5,-10$ for $N=4,7,10$, obtained from our exact solution, Eqs. (伍) (9). The exact asymptotic form, Eq. (16) is also plotted for comparison. The $N=4$ curves nearly coincide with the others, but do show some small dependence upon $T$. However, the $N=7$ and 10 curves are nearly identical and independent of $T$, and are nearly indistinguishable

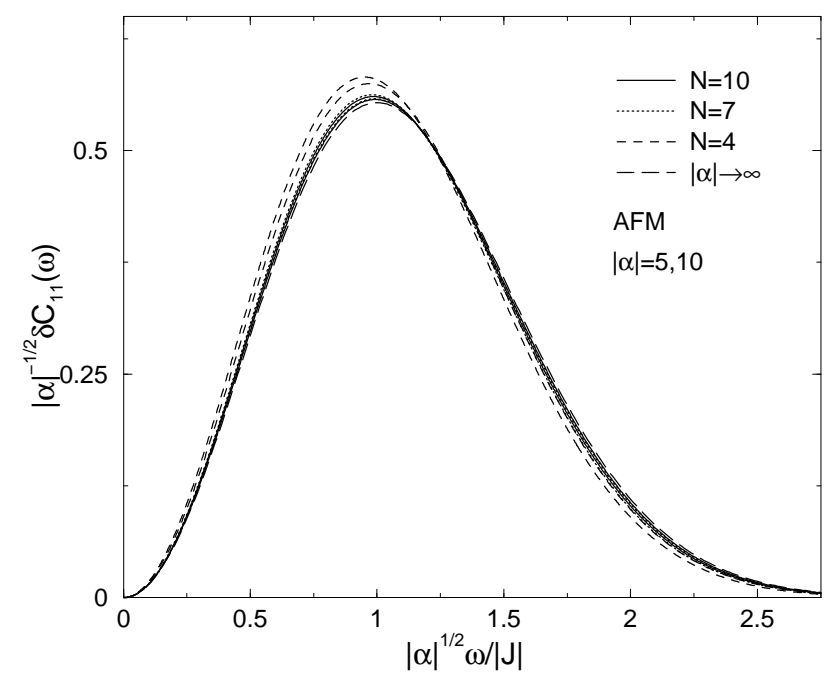

FIG. 3: Plots of the low- $T|\alpha|^{-1 / 2} \delta \mathcal{C}_{11}(\omega)$ versus $|\alpha|^{1 / 2} \omega /|J|$ for the antiferromagnetic cases with $N=4,7,10$ at $\alpha=$ $-5,-10$. Also shown is the exact $T \rightarrow 0$ limit, Eq. (16).

from the asymptotic curve for $|\alpha|=10$, when scaled in this manner.

Inverting the Fourier transform, the low- $T$ AFM $\mathcal{C}_{11}(t)$ asymptotically approaches

$$
\mathcal{C}_{11}(t) \underset{T \rightarrow 0}{\sim}\left[1+2 e^{-\bar{t}^{2}}\left(1-2 \bar{t}^{2}\right)\right] / 3,
$$

where $\bar{t}=t^{*} /\left(2|\alpha|^{1 / 2}\right)$. Hence the AFM $\lim _{T \rightarrow 0} \mathcal{C}_{11}(t)$ is a uniform function of $(J T)^{1 / 2} t$, independent of $N$. Moreover, it has precisely the same form as does $\lim _{N, T \rightarrow \infty} \mathcal{C}_{11}(t)$, Eq. (12), pictured in Fig. 1, except for the different scaling factor.

We now turn to the FM case as $T \rightarrow 0$. From Eq. (10), we expect that all of the spins will be oscillating together with frequency $N J$. But, this is strictly true only in the $T \rightarrow 0$ limit. At finite $\alpha$, the oscillation frequency deviates from this value slightly. To determine more precisely the actual nature of the mode, we note that for $\alpha>1$, the peaks in the integrand and in $Z_{N}$ both occur within $N-2 \leq s \leq N$. As $T \rightarrow 0$, we evaluate $Z_{N}$ by integration by parts, leading to the exact low- $T$ limit of the FM Fourier transform,

$$
\delta \mathcal{C}_{11}(\omega) \underset{T \rightarrow 0}{\sim} A_{N} f_{N}\left(\omega^{*}\right) / f_{N}\left(\omega_{N}^{*}\right),
$$

where $A_{N}=2 e[(N-1) / e]^{N} / N !, \omega^{*}=\omega / J$, and $\omega_{N}^{*}$, the position of the exact maximum in $f_{N}\left(\omega^{*}\right)$, is an eigenvalue of $2 \alpha s-(N-2) /(N-s)+d \ln \left[g_{0}(s)\right] / d s=0 . f_{N}$ and $g_{0}$ are given by Eqs. (5) and (6), respectively. Approximately, $\omega_{N}^{*} \approx N-(N-1) /(2 N \alpha)$, and $f_{N}\left(\omega^{*}\right)$ has a width characterized by a normal Gaussian distribution parameter $\sigma_{N}=\sqrt{N-1} /(2 N \alpha)$. Thus,

$$
\delta \mathcal{C}_{11}(\omega) \underset{\alpha \gg 1}{\approx} A_{N} \exp \left[-\left(\omega^{*}-\omega_{N}^{*}\right)^{2} /\left(2 \sigma_{N}^{2}\right)\right] .
$$




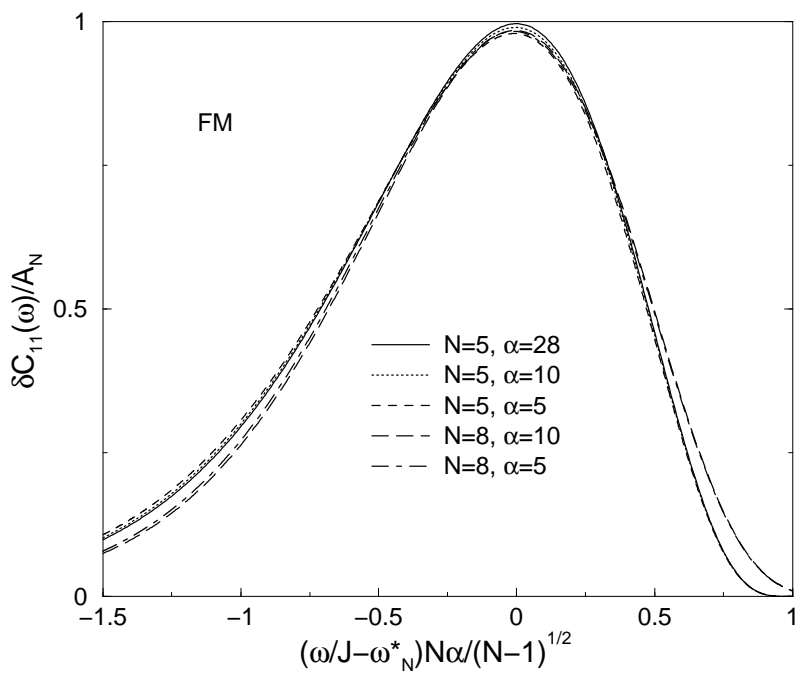

FIG. 4: Plots of the low- $T \delta \mathcal{C}_{11}(\omega) / A_{N}$ versus $(\omega / J-$ $\left.\omega_{N}^{*}\right) N \alpha / \sqrt{N-1}$, where $\omega_{N}^{*}=N-(N-1) /(2 N \alpha)$ and $A_{N}=2 e[(N-1) / e]^{N} / N$ !, for the FM cases with $N=8$, $\alpha=5,10$, and $N=5, \alpha=5,10,28$.

In this Gaussian approximation, $\omega_{N}^{*}, \sigma_{N}$, and $A_{N}$ are correctly given to their respective leading orders in $1 / \alpha$, but the skewness of the peak is not accurately represented. In Fig. 4, we have therefore plotted the scaled low- $T$ $\delta \mathcal{C}_{11}(\omega) / A_{N}$ versus $\left(\omega^{*}-\omega_{N}^{*}\right) N \alpha / \sqrt{N-1}$, for $N=8$ at $\alpha=5,10$, and for $N=5$ at $\alpha=5,10,28$, using our exact formulas, Eqs. (㺼) - (9). The $N=5$ curves nearly coincide, as do the $N=8$ curves, and the scaled height approaches 1 as $T \rightarrow 0$. As mentioned previously, the asymmetry of the curves decreases with increasing $N$, a feature not incorporated in the Gaussian approximation. Neverthess this scaling procedure works remarkably well, and $N=5$ is close to the large $N$ limit, even for the FM case.

Inverting the low-T FM Fourier transform, Eq. (19),

$$
\mathcal{C}_{11}(t) \underset{T \rightarrow 0}{\approx} 1-\frac{B_{N}}{\alpha}\left[1-e^{-t^{* 2} \sigma_{N}^{2} / 2} \cos \left(\omega_{N}^{*} t^{*}\right)\right],
$$

where $B_{N}=A_{N} \sqrt{\pi(N-1) / 2} / N$. As $N \rightarrow \infty, B_{N} \rightarrow$ $1 / N$. The deviation of the long-time limit of Eq. (20) agrees to within $1 \%$ with our results presented in Fig. 2. The decay in time of the mode in this approximation is Gaussian, with a lifetime $\tau_{N}=1 / \sigma_{N}$. Thus, as $T \rightarrow$ 0 , the oscillations are characterized by a frequency that approaches $N J$ linearly in $T$, a lifetime that diverges as $1 / T$, and an amplitude that vanishes as $T$.

Finally, we remark that our exact results for $\lim _{T \rightarrow \infty} \mathcal{C}_{11}(t)$ in the $N$-spin equivalent neighbor model may also lead to the correct $T \rightarrow \infty$ limit of the nearneighbor correlation function $\mathcal{C}_{12}(t)$ of $N$-spin rings with classical Heisenberg near-neighbor exchange coupling.
From Eq. (13) and the sum rule, we conjecture that, as for the $N$-spin equivalent neighbor model,

$$
\lim _{T \rightarrow \infty} \mathcal{C}_{12}(t)=1 / N-\delta_{N}-f_{N}(t)
$$

for the $N$-spin ring. In support of this conjecture, we have studied the $N$-spin systems consisting of $M$ spins $\mathbf{s}_{j}^{\prime}$ coupled to $N-M$ spins $\mathbf{s}_{i}$ on a ring. Letting $\mathbf{s}=$ $\sum_{i=1}^{N-M} \mathbf{s}_{i}$ and $\mathbf{s}^{\prime}=\sum_{j=1}^{M} \mathbf{s}_{j}^{\prime}$, the Hamiltonian is

$$
H=-J \sum_{i=1}^{N-M} \mathbf{s}_{i} \cdot \mathbf{s}_{i+1}-J^{\prime} \mathbf{s} \cdot \mathbf{s}^{\prime}
$$

where $\mathbf{s}_{N-M+1}=\mathbf{s}_{1}$, which is integrable. [10] Setting $\mathcal{C}^{\prime}(t)=\left\langle\mathbf{s}^{\prime}(t) \cdot \mathbf{s}^{\prime}(0)\right\rangle$, we found for $N-M=3, M=1,2,3$, and for $N-M=4, M=1,2$, that

$$
\lim _{T \rightarrow \infty} \mathcal{C}^{\prime}(t)=M^{2} / N+M(N-M)\left[\delta_{N}+f_{N}(t)\right] .
$$

Most significant of these is the result for the four-spin ring with $M=2$. We also showed previously that Eq. (21) is valid for four- and three-spin rings. [6, 7 ]

In summary, we have solved exactly for the time autocorrelation function $\mathcal{C}_{11}(t)$ of a spin in the $N$-spin equivalent neighbor model. As $T \rightarrow \infty, \mathcal{C}_{11}(t) \propto t^{-N}$ for $J t \gg 1$, curves for different $N$ exhibit very similar shapes when plotted as functions of $|J| t[(N-1)]^{1 / 2}$, and an accurate $1 / N$ asymptotic expansion is found. At low $T$, the antiferromagnetic $\mathcal{C}_{11}(t)$ is a universal function of $t T^{1 / 2}$, independent of $N$, and exhibiting strong frustration. For the ferromagnetic case at low $T$, there is a single mode with a peak position at $\approx N J-k_{B} T(N-1) / N$, and a shape that fits an accurate scaling relation. We conjecture that the $T \rightarrow \infty$ limit of the near-neighbor correlation functions $\mathcal{C}_{12}(t)$ for the $N$-spin equivalent neighbor model and for the $N$-spin ring may be identical.

* Electronic address: rklemm@mpipks-dresden.mpg.de

$\dagger$ Electronic address: marco@mpipks-dresden.mpg.de

[1] D. Gatteschi, A. Caneschi, L. Pardi, and R. Sessoli, Science 265, 1054 (1994).

[2] A. Lascialfari et al., Phys. Rev. B 55, 14341 (1997).

[3] M.H.Julien et al., Phys. Rev. Lett. 83, 227 (1999).

[4] R. Caciuffo et al., Phys. Rev. Lett. 81, 4744 (1998).

[5] M. Luban and J. Luscombe, Am. J. Phys. 67, 1161 (1999).

[6] R. Klemm and M. Luban, Phys. Rev. B 64, 104424 (2001), (see also cond-mat/0105050).

[7] M. Ameduri and R. Klemm, cond-mat/0108213.

[8] C. Kittel and H. Shore, Phys. Rev. 138, A1165 (1965).

[9] J.-M. Liu and G. Müller, Phys. Rev. B 44, 12020 (1991).

[10] N. Srivastava et al., Z. Phys. B 70, 251 (1988). 